\title{
Avoider-Enforcer star games
}

\author{
Andrzej Grzesik 1" \\ Alon Naor 非
Mirjana Mikalački $\|^{\|}$ Balázs Patkós, ${ }^{3,5}$
Zoltán Lóránt Nagy 非 Fiona Skerman $\llbracket$ \\ ${ }^{1}$ Theoretical Computer Science Dep., Fac. of Math. and Computer Science, Jagiellonian Univ., Krakow, Poland \\ ${ }^{2}$ Department of Mathematics and Informatics, Fac. of Sciences, University of Novi Sad, Serbia \\ ${ }^{3}$ Alfréd Rényi Institute of Mathematics, Budapest, Hungary \\ ${ }^{4}$ School of Mathematical Sciences, Raymond and Beverly Sackler Fac. of Exact Sciences, Tel Aviv University, Israel \\ ${ }^{5}$ MTA-ELTE Geometric and Algebraic Combinatorics Research Group, Budapest, Hungary \\ ${ }^{6}$ University of Oxford, Department of Statistics, Oxford, United Kingdom \\ received $10^{\text {th }}$ July 2013, revised $29^{\text {th }}$ Jan. 2015, accepted $9^{\text {th }}$ Mar. 2015. \\ In this paper, we study $(1: b)$ Avoider-Enforcer games played on the edge set of the complete graph on $n$ vertices. \\ For every constant $k \geq 3$ we analyse the $k$-star game, where Avoider tries to avoid claiming $k$ edges incident to \\ the same vertex. We consider both versions of Avoider-Enforcer games - the strict and the monotone - and for each \\ provide explicit winning strategies for both players. We determine the order of magnitude of the threshold biases \\ $f_{\mathcal{F}}^{\text {mon }}, f_{\mathcal{F}}^{-}$and $f_{\mathcal{F}}^{+}$, where $\mathcal{F}$ is the hypergraph of the game.
}

Keywords: positional games, complete graph, Avoider-Enforcer

\section{Introduction}

Let $a$ and $b$ be two positive integers, let $X$ be a finite set and let $\mathcal{F} \subseteq 2^{X}$ be a family of subsets of $X$. In an $(a: b)$ Avoider-Enforcer game $\mathcal{F}$, two players, called Avoider and Enforcer, alternately claim $a$ and $b$ previously unclaimed elements of $X$ per move, respectively. If the number of unclaimed elements is strictly less than $a$ (respectively $b$ ) before Avoider's (respectively Enforcer's) move, then he claims all these elements. The game ends when all the elements of $X$ have been claimed by either of the players. Avoider loses the game if by the end of the game he has claimed all the elements of some $F \in \mathcal{F}$, and

\footnotetext{
*Email: andrzej.grzesikeuj.edu.pl.

${ }^{\dagger}$ Email: mirjana.mikalacki@dmi.uns.ac.rs. Research partly supported by Ministry of Education and Science, Republic of Serbia, and Provincial Secretariat for Science, Province of Vojvodina.

‡Email: nagy.zoltan.loranterenyi.mta.hu. Supported by Hungarian National Scientific Research Funds (OTKA) grant 81310.

§Email: alonnaorepost.tau.ac.il.

IEmail: patkosbecs.elte.hu and patkos@renyi.hu. Research supported by the János Bolyai Research Scholarship of the Hungarian Academy of Sciences.

"Email: skerman@stats.ox.ac.uk.

1365-8050 @ 2015 Discrete Mathematics and Theoretical Computer Science (DMTCS), Nancy, France
} 
wins otherwise. Throughout this paper we assume that Avoider is the first player to play, although usually it makes very little difference. We refer to $X$ as the board of the game, to $\mathcal{F}$ as the target sets, and to $a$ and $b$ as the bias of Avoider and Enforcer, respectively. Since the pair $(X, \mathcal{F})$ is a hypergraph that represents the game, we often refer to $\mathcal{F}$ as the hypergraph of the game, or as the game itself.

Avoider-Enforcer games are the misère version of the well-studied Maker-Breaker games. In an $(a: b)$ Maker-Breaker game $\mathcal{F}$, the two players are called Maker and Breaker, they claim respectively $a$ and $b$ elements of $X$ per move, and Maker wins if and only if by the end of the game he has claimed all the elements of some $F \in \mathcal{F}$. Both Maker-Breaker and Avoider-Enforcer games are finite, perfect information games, and there is no possibility of a draw. Hence, for every given setup $-a, b, \mathcal{F}-$ one of the players has a winning strategy. We say that this player wins the game.

It is very natural to play both Avoider-Enforcer and Maker-Breaker games on the edge set of a given graph $G$, and specifically for $G=K_{n}$, the complete graph on $n$ vertices. In this case the board is $X=E\left(K_{n}\right)$ and the target sets are $\mathcal{F} \subseteq 2^{E\left(K_{n}\right)}$. For example: in the connectivity game $\mathcal{C}_{n}$ the target sets are all edge sets of connected graphs on $n$ vertices; in the perfect matching game $\mathcal{M}_{n}$ the target sets are all graphs on $n$ vertices containing a perfect matching (we assume $n$ is even here); in the Hamiltonicity game $\mathcal{H}_{n}$ the target sets are all edge sets of graphs on $n$ vertices containing a Hamilton cycle. We usually omit the subindex $n$ in our notation. These three games were initially studied in Maker-Breaker version by Chvátal and Erdős in their seminal paper [4].

Many natural games played on the edges of $K_{n}$ (including all the above mentioned ones) are drastically in favor of Maker, i.e. Maker wins in the unbiased $(1: 1)$ version in (almost) minimal number of moves required to create a winning set. Therefore, it makes sense to give more power to Breaker in order to even out the odds, and typically the $(1: b)$ version is considered. In addition, Maker-Breaker games are bias monotone: if Maker wins some game $\mathcal{F}$ with bias $(a: b)$, he also wins this game with bias $\left(a^{\prime}: b^{\prime}\right)$, for every $a^{\prime} \geq a$ and $b^{\prime} \leq b$. This bias monotonicity enables the definition of the threshold bias: for a given hypergraph $\mathcal{F}$, the threshold bias $f_{\mathcal{F}}$ is the unique integer for which Maker wins the $(1: b)$ game $\mathcal{F}$ for every $b<f_{\mathcal{F}}$, and Breaker wins the $(1: b)$ game $\mathcal{F}$ for every $b \geq f_{\mathcal{F}}$.

Unfortunately, Avoider-Enforcer games are not bias monotone in general (see e.g. [6], [7]): although intuitively each player wishes to claim as few elements as possible, it is sometimes a disadvantage to claim fewer elements per move, for any of the players. This makes the analysis of these games much more difficult, and it is not possible to define the threshold bias in the same manner as in Maker-Breaker games. Therefore, Hefetz, Krivelevich and Szabó introduced in [7] the following parameters. The lower threshold bias $f_{\mathcal{F}}^{-}$is the largest integer such that Enforcer wins the $(1: b)$ game $\mathcal{F}$ for every $b \leq f_{\mathcal{F}}^{-}$. The upper threshold bias $f_{\mathcal{F}}^{+}$is the smallest non-negative integer such that Avoider wins the $(1: b)$ game $\mathcal{F}$ for every $b>f_{\mathcal{F}}^{+}$. Except for some trivial cases, $f_{\mathcal{F}}^{-}$and $f_{\mathcal{F}}^{+}$always exist and satisfy $f_{\mathcal{F}}^{-} \leq f_{\mathcal{F}}^{+}$. When $f_{\mathcal{F}}^{-}=f_{\mathcal{F}}^{+}$we call this number $f_{\mathcal{F}}$ and refer to it as the threshold bias of the game $\mathcal{F}$.

In order to overcome this bias monotonicity obstacle, Hefetz, Krivelevich, Stojaković and Szabó proposed in [6] a bias monotone version for Avoider-Enforcer games: they suggested that Avoider and Enforcer will claim at least $a$ and $b$ board elements per move, respectively. It is easy to see that this new version is indeed bias monotone, i.e. each player can only benefit from lowering his bias. This fact allowed them to define for any given hypergraph $\mathcal{F}$ the monotone threshold bias $f_{\mathcal{F}}^{m o n}$ as the largest non-negative integer for which Enforcer wins the $(1: b)$ game $\mathcal{F}$ under the new set of rules if and only if $b \leq f_{\mathcal{F}}^{m o n}$. Throughout this paper we refer to this new set of rules as the monotone rules, to distinguish it from the strict rules. Accordingly, we refer to the games played under the two sets of rules as monotone games and as strict games, respectively. 
Interestingly, these seemingly minor adjustments in the rules can completely change the outcome of the game. For example, even in such a natural game as the connectivity game, the two versions of the game are essentially different. In [7] it was shown that Avoider wins the strict $(1: b)$ connectivity game played on $E\left(K_{n}\right)$ if and only if at the end of the game he has at most $n-2$ edges, therefore the threshold bias exists and is of linear order. On the other hand, the monotone threshold bias for this game is of order $\frac{n}{\ln n}$ [6. 9].

Naturally, one may ask about the relationship between $f_{\mathcal{F}}^{-}, f_{\mathcal{F}}^{+}$and $f_{\mathcal{F}}^{m o n}$. Specifically, it could be expected that $f_{\mathcal{F}}^{-} \leq f_{\mathcal{F}}^{m o n} \leq f_{\mathcal{F}}^{+}$holds for every family $\mathcal{F}$. The above mentioned connectivity game shows that this is not true in general, even when there exists a threshold bias in the strict game.

In [7], Hefetz, Krivelevich and Szabó provided a general sufficient condition for Avoider's win in $(a: b)$ Avoider-Enforcer games played under both sets of rules. This criterion takes only Avoider's bias into account. In [2], Bednarska-Bzdȩga introduced a new sufficient condition for Avoider's win under both sets of rules, which depends on both parameters $a$ and $b$, and gives a better result than the one in [7] in cases where the hypergraph of the game has rank smaller than $b$.

In [6], Hefetz et al. investigated $(1: b)$ Avoider-Enforcer games played on the edge set of $K_{n}$, where Avoider wants to avoid claiming a copy of some fixed graph $H$. In this case $X=E\left(K_{n}\right)$, and $\mathcal{F}=$ $\mathcal{K}_{H} \subseteq 2^{E\left(K_{n}\right)}$ consists of all subgraphs of $K_{n}$ containing $H$ as a subgraph. These games are referred to as $H$-games. They conjectured that for any fixed graph $H$, the thresholds $f_{\mathcal{K}_{H}}^{-}$and $f_{\mathcal{K}_{H}}^{+}$are not of the same order of magnitude, and wondered about the connection between monotone $H$-games and strict $H^{-}$-games, where $H^{-}$is $H$ with one edge missing. They investigated $H$-games where $H=K_{3}$ (a triangle) and $H=P_{3}$ (a path on three vertices) and established the following:

$$
f_{\mathcal{K}_{P_{3}}}^{\text {mon }}=\left(\begin{array}{l}
n \\
2
\end{array}\right)-\left\lfloor\frac{n}{2}\right\rfloor-1, \quad f_{\mathcal{K}_{P_{3}}}^{+}=\left(\begin{array}{l}
n \\
2
\end{array}\right)-2, \quad f_{\mathcal{K}_{P_{3}}}^{-}=\Theta\left(n^{\frac{3}{2}}\right) \quad \text { and } \quad f_{\mathcal{K}_{K_{3}}}^{\text {mon }}=\Theta\left(n^{\frac{3}{2}}\right) .
$$

They used this example to support their conjecture, as $f_{\mathcal{K}_{P_{3}}}^{+}$and $f_{\mathcal{K}_{P_{3}}}^{-}$are indeed not of the same order. They also noted that $f_{\mathcal{K}_{K_{3}}}^{\text {mon }}$ and $f_{\mathcal{K}_{P_{3}}}^{-}$are of the same order, and that $P_{3}=K_{3}^{-}$. Bednarska-Bzdęga established in [2] general upper and lower bounds on $f_{\mathcal{K}_{H}}^{+}, f_{\mathcal{K}_{H}}^{-}$and $f_{\mathcal{K}_{H}}^{\text {mon }}$ for every fixed graph $H$, but these bounds are not tight for every graph $H$. In order to prove our main result of this paper we prove a number theoretic fact, which we later use independently in order to improve one of Bednarska-Bzdȩga's bounds. We elaborate on that in Section 5 .

Our main objective in this paper is to study monotone and strict $H$-games played on the edges of $K_{n}$, where $H$ is the $k$-star $K_{1, k}$, denoted by $\mathcal{S}_{k}$, for any fixed $k \geq 3$. We refer to this game as the star game, or more specifically, for a given $k$, we call this game the $k$-star game. Studying the star game is very natural, since avoiding a $k$-star in Avoider's graph is exactly keeping its maximal degree strictly below $k$. We analyse this game, provide explicit winning strategies for both players under both sets of rules, and obtain the following.

Theorem 1.1. For every $k \geq 3$ and for every large enough $n$ the following bounds hold:
(i) $\frac{2}{5} n^{\frac{k}{k-1}} \leq f_{\mathcal{K}_{\mathcal{S}_{k}}^{m o n}} \leq 2 n^{\frac{k}{k-1}}$;
(ii) $\frac{1}{5} n^{\frac{k}{k-1}} \leq f_{\mathcal{K}_{\mathcal{S}_{k}}}^{+} \leq 2 n^{\frac{k}{k-1}}$;
(iii) $\frac{1}{2} n^{\frac{k+1}{k}} \leq f_{\mathcal{K}_{\mathcal{S}_{k}}} \leq 2 n^{\frac{k+1}{k}}$. 
These results show that $f_{\mathcal{K}_{\mathcal{S}_{k}}}$ and $f_{\mathcal{K}_{\mathcal{S}_{k}}}^{+}$are not of the same order for any given $k \geq 3$, supporting the conjecture of Hefetz et al. from [6]. In addition, as $\mathcal{S}_{k}^{-}=\mathcal{S}_{k-1}$, an immediate consequence of Theorem 1.1 is that $f_{\mathcal{K}_{\mathcal{S}_{k}}}^{m o n}$ and $f_{\mathcal{K}_{\mathcal{S}_{k}^{-}}}$are of the same order, showing a strong connection between the monotone $H$-game and the strict $H^{-}$-game in this case. Note that $\mathcal{S}_{2}=P_{3}$, so the $k$-star game for $k=2$ is already covered in [6]. In fact, the results there match ours, if we generalize Theorem 1.1 to include the case $k=2$. However, since these results are known, and in order to avoid some technical difficulties in our proofs, we only consider the case $k \geq 3$.

The outcome of some $(1: b)$ positional games played on the edges of $K_{n}$, where the target sets possess some graph property $\mathcal{P}$, is the same as in the corresponding games where the players play randomly. This phenomenon was first observed by Chvatál and Erdős in [4] for the Maker-Breaker connectivity game, and is known as the random graph intuition. The reason for this name is that when both players play randomly the $(1: b)$ game, the graph of the player with bias 1 (either Maker or Avoider) at the end of the game satisfies $G \sim G(n, m)$, where $m=\left\lceil\frac{1}{b+1}\left(\begin{array}{l}n \\ 2\end{array}\right)\right\rceil$. For this given $m$, the graph $G(n, m)$ behaves in many ways similarly to $G\left(n, \frac{1}{b+1}\right)$, the random graph on $n$ vertices where each potential edge appears in the graph independently with probability $\frac{1}{b+1}[8]$. In other words, the threshold bias $b^{*}$ for these games is asymptotically equal to $1 / p^{*}$, where $p^{*}$ is the threshold probability for the appearance of $\mathcal{P}$ in $G \sim G(n, p)$.

The $k$-star game is a very good example for this phenomenon, as indeed the properties of the random graph $G \sim G\left(n, \frac{1}{b+1}\right)$ suggest the outcome of the Avoider-Enforcer $(1: b) k$-star game. All the following statements about $G$ hold w.h.p. (i.e. with probability tending to 1 as $n$ tends to infinity). For details the reader may refer to [3], Theorem 3.1.

- For $b=\omega\left(n^{\frac{k}{k-1}}\right)$ the maximal degree in $G$ is at most $k-2$, and Avoider wins the $(1: b)$ game (both strict and monotone).

- At $b=\Theta\left(n^{\frac{k}{k-1}}\right)$, vertices of degree $k-1$ emerge in $G$. If Avoider claims the last edge in the $(1: b)$ game, the appearance of a vertex of degree $k-1$ in his graph before the last round means he loses, and this is indeed the order of magnitude of $f_{\mathcal{S}_{k}}^{m o n}$ and $f_{\mathcal{S}_{k}}^{+}$, where presumably Avoider claims the last edge.

- When $b=\omega\left(n^{\frac{k+1}{k}}\right)$ and $b=o\left(n^{\frac{k}{k-1}}\right)$, the maximal degree in $G$ is exactly $k-1$. The outcome of the strict $(1: b)$ game heavily depends on the number of free edges Avoider will be able to choose from in his last move, and so the outcome oscillates.

- Finally, for $b \leq C n^{\frac{k+1}{k}}$, where $C$ is a sufficiently small constant, vertices of degree $k$ emerge in $G$, and Enforcer wins the $(1: b)$ game (both strict and monotone).

The rest of the paper is organized as follows: in Section 3 we provide Avoider's strategy for the $k$-star game which applies for both versions of the game. In Section 4 we provide Enforcer's strategies for the $k$-star game, one strategy for the monotone game and one for the strict game. In Section 5 we improve one of Bednarska-Bzdęga's bounds for general $H$-games. Finally, in Section 6 we present some concluding remarks and open problems. 


\section{Preliminaries}

Throughout this paper we use the following notation.

A previously unclaimed edge is called a free edge. The act of claiming one free edge by one of the players is called a step. In the strict game, Enforcer's $b$ (respectively Avoider's 1) successive steps are called a move. In the monotone game, each move consists of at least $b$ steps, respectively at least one step. A round in the game consists of one move of the first player (Avoider), followed by one move of the second player (Enforcer). Whenever one of the players claims an edge incident to some vertex $u$, we say that the player touched $u$.

Our graph-theoretic notation is standard and follows that of [10]. In particular, throughout the paper $G$ stands for a simple graph with vertex set $V=V(G)$ and edge set $E=E(G)$. For any subset $U \subseteq V$ we say that an edge $u v$ lies inside $U$ if $u, v \in U$. For $i \geq 0$, we denote by $A_{i}$ and $E_{i}$ the graphs with vertex set $V$, whose edges were claimed by Avoider, respectively Enforcer, in the first $i$ rounds. For every vertex $v \in V$ and every $i \geq 0$, let $d_{A_{i}}(v)$ and $d_{E_{i}}(v)$ denote the degree of $v$ in $A_{i}$, respectively $E_{i}$. We sometimes omit the subindex $i$ when its value is clear or irrelevant. In these cases we also refer to $d_{A}(v)$ as the $A$-degree of $v$. Whenever we consider the end of the $i$ th round for the case $i=0$, we simply refer to the beginning of the game, before any move was played.

The set of all free edges at the end of the $i$ th round is denoted by $F_{i}$. A free edge is called a threat if it is incident to a vertex of $A$-degree $k-1$.

For the sake of simplicity and clarity of presentation, no real effort has been made here to optimize the constants appearing in our results. We also omit floor and ceiling signs whenever these are not crucial. Our results are asymptotic in nature and whenever necessary we assume that $n$ is sufficiently large. We use $o(1)$ to denote a positive function of $n$, tending to zero as $n$ tends to infinity.

For every two integers $n$ and $b$ let $r=r(n, b)$ be the integer for which $1 \leq r \leq b+1$ and $\left(\begin{array}{c}n \\ 2\end{array}\right) \equiv r \bmod$ $(b+1)$ hold. The value of $r$ is the number of free edges before the last round of the strict game, and since Avoider is the first player, $r$ is actually the number of edges which remain for Avoider to choose from in his last move. Therefore, this value may be very significant in determining the identity of the winner in the strict game. In order to estimate $r$ in some cases, we need the following two number theoretical statements.

Fact 2.1. Let $c$ and $\alpha$ be two constants such that either $\alpha=1$ and $c \geq 1$, or $\alpha \in(1,2)$ and $c>0$. For any sufficiently large integer $n$ there exists an integer $q=(2-o(1)) c n^{\alpha}$ such that the remainder of the division of $\left(\begin{array}{l}n \\ 2\end{array}\right)$ by $q$ is larger than $c n^{\alpha}$.

Proof: Let $N=\left(\begin{array}{c}n \\ 2\end{array}\right), N^{\prime}=N-c n^{\alpha}-1, m=\left\lceil\frac{1}{4 c} n^{2-\alpha}\right\rceil$ and $q=\left\lfloor\frac{N^{\prime}}{m}\right\rfloor$. Note that $N^{\prime}=q m+r$ for some $0 \leq r<m$ and that $q=(2-o(1)) c n^{\alpha}$. Since $N-q m=c n^{\alpha}+r+1<q$, it follows that the remainder of the division of $N$ by $q$ is larger than $c n^{\alpha}$.

Fact 2.2. For every sufficiently large integer $n$ and for every constant $k \geq 3$ there exists a $c=c(n, k)$ such that $\frac{1}{5}<c<\frac{1}{4}$ and the remainder $r$ of the division of $\left(\begin{array}{l}n \\ 2\end{array}\right)$ by $c n^{\frac{k}{k-1}}$ is positive and satisfies $r=o(n)$.

Proof: Let $N=\left(\begin{array}{c}n \\ 2\end{array}\right)$ and let $M=\left\{2\left\lceil n^{1-\frac{1}{k-1}}\right\rceil+i: 0 \leq i \leq 4\right\}$. The least common multiple of the elements in $M$ is at least $n^{2.5}$ so there exists an element $m \in M$ which does not divide $N$. Let $q=\left\lfloor\frac{N}{m}\right\rfloor$ and note that $q=\left(\frac{1}{4}-o(1)\right) n^{\frac{k}{k-1}}$. Since $N-m<q m<N$, the remainder $r$ of the division of $N$ by $q$ satisfies $0<r<m=o(n)$. 


\section{Avoider's strategy}

In this section we establish upper bounds on the threshold biases $f_{\mathcal{K}_{\mathcal{S}_{k}}}^{\text {mon }}, f_{\mathcal{K}_{\mathcal{S}_{k}}}^{+}$and $f_{\mathcal{K}_{\mathcal{S}_{k}}}^{-}$.

We provide Avoider with the following trivial strategy $\mathcal{S}_{A}$ : in every move Avoider claims one arbitrary edge which does not increase the maximal degree in his graph if such an edge exists, and an arbitrary edge otherwise. Clearly Avoider can follow this strategy. Note that this is a valid strategy for both the monotone and the strict versions of the game.

Consider the course of a game (either strict or monotone) in which Avoider plays according to $\mathcal{S}_{A}$ and Enforcer plays according to some fixed strategy. For every $i$, let $I_{i}$ denote the set of vertices of maximal $A$ degree at the end of round $i$. Let $s$ be the maximal $A$-degree at the end of the game. For every $0 \leq j \leq s$, let $i_{j}$ be the largest integer such that the maximal $A$-degree at the end of round $i_{j}$ is $j$. Note that the maximal $A$-degree is never increased by more than one according to $\mathcal{S}_{A}$, and so $0=i_{0}<i_{1}<\cdots<i_{s}$.

Lemma 3.1. $\left|F_{i_{j}}\right| \leq\left(2^{j-1}+o(1)\right) \frac{n^{j+2}}{b^{j}}$ for every $0 \leq j \leq s$.

Proof: Observe that at the end of round $i_{j}$ (for every $j$ ) every free edge has at least one endpoint in $I_{i_{j}}$, as otherwise Avoider will not increase the maximal degree in his graph in his subsequent move. Therefore, if $\left|I_{i_{j}}\right| \leq\left(2^{j-1}+o(1)\right) \frac{n^{j+1}}{b^{j}}$ then $\left|F_{i_{j}}\right| \leq\left|I_{i_{j}}\right| \cdot n \leq\left(2^{j-1}+o(1)\right) \frac{n^{j+2}}{b^{j}}$. Since the number of free edges at the beginning of the game is obviously $\left(\begin{array}{l}n \\ 2\end{array}\right) \leq 2^{-1} n^{2}$, it suffices to show that if $\left|F_{i_{j}}\right| \leq\left(2^{j-1}+o(1)\right) \frac{n^{j+2}}{b^{j}}$, then $\left|I_{i_{j+1}}\right| \leq\left(2^{j}+o(1)\right) \frac{n^{j+2}}{b^{j+1}}$, for every $0 \leq j<s$.

Indeed, as both players claim altogether at least $b+1$ edges, for every $0 \leq j<s$ the number of rounds in the game after round $i_{j}$ cannot be greater than $\left\lceil\left|F_{i_{j}}\right| /(b+1)\right\rceil \leq\left(2^{j-1}+o(1)\right) \frac{n^{j+2}}{b^{j+1}}$. Since Avoider claims exactly one edge per move, in each round after round $i_{j}$ at most two new vertices of $A$-degree $j+1$ appear. Therefore, $\left|I_{i_{j+1}}\right| \leq\left(2^{j}+o(1)\right) \frac{n^{j+2}}{b^{j+1}}$.

Now it is easy to see that $\mathcal{S}_{A}$ is a winning strategy for Avoider in the $(1: b)$ Avoider-Enforcer $k$-star game for any $b \geq 2 n^{\frac{k}{k-1}}$ and under both sets of rules, thus obtaining the upper bounds in Theorem $1.1(i)$ and $(i i)$. Indeed, it follows by Lemma 3.1 that if $i_{k-2}$ exists then $\left|F_{i_{k-2}}\right| \leq n^{\frac{k}{k-1}}<b$. Therefore no threat appears before Avoider's last move and so he wins.

We now prove the upper bound for $f_{\mathcal{K}_{\mathcal{S}_{k}}}^{-}$given in Theorem 1.1 (iii). By Fact 2.1. with $c=1$ and $\alpha=\frac{k+1}{k}$, there exists an integer $b=(2-o(1)) n^{\frac{k+1}{k}}$ such that $r(n, b)>n^{\frac{k+1}{k}}$. Assume that $s \geq k-1$ for this $b$ (otherwise Avoider obviously wins). It follows by Lemma 3.1 that $\left|F_{i_{k-1}}\right|<n^{\frac{k+1}{k}}$. However, by the assumption on $r$ there are more free edges than $n^{\frac{k+1}{k}}$ before any move of Avoider, so $i_{k-1}$ is the last round of the game, meaning $s=k-1$.

Remark 3.2. All arguments in this section are still valid even if we include the case $k=2$.

\section{Enforcer's strategies}

In this section we establish the lower bounds given in Theorem 1.1 Unlike Avoider's strategy, which was valid for both versions of the game, here we distinguish between the two cases. We start with the monotone game which is simpler to analyse and establish the lower bound on $f_{\mathcal{K}_{\mathcal{S}_{k}}}^{\text {mon }}$. Then we proceed to the strict game, explain the adjustments we make to Enforcer's strategy and establish the lower bounds on $f_{\mathcal{K}_{\mathcal{S}_{k}}}^{+}$and $f_{\mathcal{K}_{\mathcal{S}_{k}}}$. 


\subsection{The monotone game}

We provide a strategy for Enforcer for the monotone $(1: b) k$-star game for $b=\frac{2}{5} n^{\frac{k}{k-1}}$. At any point during the game, let $I$ denote the set of isolated vertices in Enforcer's graph, and let $C=V \backslash I$. Furthermore, let $I_{i}$ and $C_{i}$ denote the respective sets of vertices at the end of the $i$ th round. Initially, of course, $I_{0}=V$ and $C_{0}=\emptyset$. Whenever Enforcer touches a vertex previously isolated in his graph, we say that he moved that vertex from $I$ to $C$.

For every $i \geq 0$, Enforcer plays his $(i+1)$ st move as follows.

(1) If there exists a vertex of $A$-degree at least $k$, or if there are at most $b$ free edges remaining, Enforcer claims all free edges on the board. We refer to this move as the trivial move.

(2) Otherwise, if there exists a vertex $v \in I$ of $A$-degree $k-1$, then Enforcer claims all free edges on the board, except one, incident to $v$. We refer to this move as the end move.

(3) Otherwise, let $v_{1}^{(i)}, \ldots, v_{\left|I_{i}\right|}^{(i)}$ be an enumeration of the vertices in $I_{i}$ such that for every $1 \leq j<\left|I_{i}\right|$, $d_{A_{i+1}}\left(v_{j}^{(i)}\right) \leq d_{A_{i+1}}\left(v_{j+1}^{(i)}\right)$. Let $I_{i, j}=\left\{v_{1}^{(i)}, \ldots, v_{j}^{(i)}\right\}$ and let $s_{i}$ be the smallest integer such that the number of free edges inside $C_{i} \cup I_{i, s_{i}}$ is at least $b$. Enforcer claims all the free edges inside $C_{i} \cup I_{i, s_{i}}$. We refer to this move as the base move.

We have to show that Enforcer can follow the proposed strategy, and that by doing so he wins the game. Starting with the former, it is evident that Enforcer can play the trivial move; Enforcer can play the end move since there are more than $b$ free edges on the board, and since there are $n-k$ free edges incident to $v$; finally, Enforcer can play the base move since $C_{i} \cup I_{i, s_{i}}=V$ for $s_{i}=\left|I_{i}\right|$ and there are more than $b$ free edges on the board.

We now prove that the proposed strategy is indeed a winning strategy for Enforcer. Consider the course of the game in which Enforcer plays according to the proposed strategy and Avoider plays according to some fixed arbitrary strategy. If at any point during the game the maximal $A$-degree in the graph increases to at least $k$ then Enforcer wins. He also wins if he plays the end move at some point. So assume for contradiction that neither of these events happen. Therefore, by the description of his strategy, it is clear that Enforcer plays the base move for $l$ rounds, for some $l \geq 0$, and then either the game ends or in his last move he plays the trivial move since there are at most $b$ free edges remaining.

Observation 4.1. Throughout the game, the following properties hold.

(i) There are at least $n-k$ free edges incident to every vertex in I.

(ii) After every move, by either player, every free edge has at least one endpoint in I.

(iii) The number of edges claimed by both players in each round of the game is at most $(1+o(1)) b$.

Proof:

(i) This is obvious since every vertex in $I$ is isolated in Enforcer's graph and every vertex has $A$-degree less than $k$. 
(ii) The claim is true after each base move played by Enforcer by his strategy, and there are no free edges left after he plays the trivial move, if he does. Recall that by assumption he never plays the end move. In addition, Avoider does not change the set $I$, so the claim remains true after his moves as well.

(iii) Whenever Enforcer plays the base move he does not claim more than $b+n=(1+o(1)) b$ edges. If he plays the trivial move this is obviously still true. Finally, Avoider claims at most $\frac{k n}{2}=o(b)$ edges throughout the game, otherwise a vertex of $A$-degree at least $k$ must exist.

Now we wish to estimate the $A$-degrees of vertices in $I$. Let $T(i):=\frac{1}{\left|I_{i}\right|} \sum_{v \in I_{i}} d_{A_{i}}(v)$ denote the average $A$-degree of the vertices in $I_{i}$ at the end of round $i$. Note that by definition $T(0)=0$, and that $T(i)>T(i-1)$ if Enforcer plays the base move in his $i$ th move. Indeed, since throughout the game all free edges have at least one endpoint in $I$, Avoider in his $i$ th move increases the sum of $A$-degrees in $I$ (while not changing the set itself), and Enforcer in his subsequent move removes from $I$ vertices of minimal $A$-degree, so he does not decrease the average $A$-degree in $I$.

Claim 4.2. For every $0 \leq j \leq k-2$, the following holds. If $0<\left|I_{i}\right|<\frac{9}{10} n^{1-\frac{j}{k-1}}$ for some $i$, then $T(i) \geq j$.

Proof: We prove the claim by induction on $j$. The claim trivially holds for $j=0$, as $T(i) \geq 0$ for every $i$. Suppose now for contradiction that for some $1 \leq j \leq k-2$, the claim holds for $j-1$, but not for $j$. Then there exists an integer $i$ such that $0<\left|I_{i}\right|<\frac{9}{10} n^{1-\frac{j}{k-1}}$, but $T(i)<j$, which implies $\sum_{v \in I_{i}} d_{A_{i}}(v)<$ $j\left|I_{i}\right|$. Let $i_{0} \leq i$ be the minimal index such that $T\left(i_{0}\right) \geq j-1$ (by the induction hypothesis and the size of $I_{i}$, such an index exists), and for every $i_{0} \leq s \leq i$ let $W(s):=\sum_{v \in I_{s}}\left(d_{A_{s}}(v)-(j-1)\right)$. Note that $W(i)<\left|I_{i}\right|$ by the assumption on the index $i$.

Since $T(i)<j$, there are vertices of $A$-degree less than $j$ in $I_{i}$, and therefore, according to his strategy, Enforcer has only moved vertices of $A$-degree less than $j$ from $I$ to $C$ in his first $i$ moves. In addition, Avoider increases the sum of $A$-degrees of the vertices in $I$ in each of his moves. It follows that $W(s+$ $1)>W(s)$ for every $i_{0} \leq s<i$. Since $W\left(i_{0}\right) \geq 0$ by definition of $i_{0}$, and since $W(s)$ is an integer for every $s$, we get that $i-i_{0}+1 \leq W(i)+1 \leq\left|I_{i}\right|<\frac{9}{10} n^{1-\frac{j}{k-1}}$. It follows that between rounds $i_{0}$ and $i$, including round $i_{0}$ if $i_{0}>0$, Enforcer has claimed at most $(1+o(1)) b\left|I_{i}\right|<\frac{2}{5} n^{2-\frac{j-1}{k-1}}$ edges.

On the other hand, consider the vertices that were moved from $I$ to $C$ by Enforcer between rounds $i_{0}$ and $i$. Let $I^{*}=I_{i_{0}-1}$ if $i_{0}>0$, and $I^{*}=I_{0}=V$ otherwise (note that $i_{0}=0$ if and only if $j=1$ ). Since throughout the game every vertex in $I$ has at least $n-k$ free edges incident to it, and by using the induction hypothesis, we conclude that during the specified rounds Enforcer must have claimed at least

$$
\begin{aligned}
\frac{\left(\left|I^{*}\right|-\left|I_{i}\right|\right)\left(\left|C_{i}\right|-k\right)}{2} & \geq(1-o(1))\left(\frac{9}{10} n^{1-\frac{j-1}{k-1}}-\frac{9}{10} n^{1-\frac{j}{k-1}}\right) \frac{\left|C_{i}\right|}{2} \\
& =(1-o(1)) \frac{9}{20} n^{2-\frac{j-1}{k-1}} \\
& >\frac{2}{5} n^{2-\frac{j-1}{k-1}}
\end{aligned}
$$

edges, a contradiction. 
Let $i$ be the maximal index such that $\left|I_{i}\right|>0$ and $T(i)<k-2$ (there exists such an index since both inequalities hold for $i=0$ ). By Claim 4.2 we get $\left|I_{i}\right| \geq \frac{9}{10} n^{\frac{1}{k-1}}$. Hence, either $\left|I_{i}\right| \geq \frac{n}{1000}$ and then $\left|F_{i}\right|=\Theta\left(n^{2}\right)=\omega(b)$, or

$$
\left|F_{i}\right| \geq\left|I_{i}\right|(n-k)-\left(\begin{array}{c}
\left|I_{i}\right| \\
2
\end{array}\right) \geq\left(1-\frac{1}{100}\right)\left|I_{i}\right| n>\frac{89}{100} n^{1+\frac{1}{k-1}}>\frac{22}{10} b .
$$

Therefore, $\left|F_{i+1}\right|>\frac{11}{10} b$ by Part (iii) of Observation 4.1. and $T(i+1) \geq k-2$ by definition of $i$. Hence, after Avoider's $(i+2)$ nd move, either there exists a vertex of $A$-degree at least $k$, or there exists a vertex $v \in I$ with $A$-degree $k-1$, while there are still more than $b$ free edges on the board, in which case Enforcer plays the end move. In either case, this is a contradiction to the assumption on Avoider's strategy. This completes the proof.

\subsection{The strict game}

Recall that $r=r(n, b)$ denotes the integer which satisfies $1 \leq r \leq b+1$ and $\left(\begin{array}{l}n \\ 2\end{array}\right) \equiv r \bmod (b+1)$, i.e. the number of free edges at the beginning of the last round of the game. Let

$$
\begin{aligned}
& b_{n, k}^{+}=\max \left\{b \leq \frac{1}{4} n^{\frac{k}{k-1}}: r(n, b) \leq \frac{5}{8} \frac{n^{k+1}}{(2 b)^{k-1}}\right\}, \text { and } \\
& b_{n, k}^{-}=\max \left\{b \leq \frac{1}{4} n^{\frac{k}{k-1}}: r\left(n, b^{\prime}\right) \leq \frac{5}{8} \frac{n^{k+1}}{\left(2 b^{\prime}\right)^{k-1}} \text { for every } 1 \leq b^{\prime} \leq b\right\} .
\end{aligned}
$$

Claim 4.3. For every sufficiently large integer $n$ and for every integer $k \geq 3$ the following bounds hold:

(i) $b_{n, k}^{+} \geq \frac{1}{5} n^{\frac{k}{k-1}}$;

(ii) $b_{n, k}^{-} \geq \frac{1}{2} n^{\frac{k+1}{k}}$.

\section{Proof:}

(i) By Fact 2.2 there exists an integer $\frac{1}{5} n^{\frac{k}{k-1}} \leq b \leq \frac{1}{4} n^{\frac{k}{k-1}}$ such that $r(n, b)=o(n)$, and since $\frac{5}{8} \frac{n^{k+1}}{(2 b)^{k-1}}=\Theta(n)$ in this case, the desired inequality holds.

(ii) Note that

$$
b \leq \frac{1}{2} n^{\frac{k+1}{k}} \Longrightarrow(2 b)^{k} \leq n^{k+1} \Longrightarrow b \leq \frac{1}{2} \frac{n^{k+1}}{(2 b)^{k-1}},
$$

and since $r \leq b+1$ trivially holds, we get $b_{n, k}^{-} \geq \frac{1}{2} n^{\frac{k+1}{k}}$.

The lower bounds in Theorem 1.1 (ii) and (iii) follow directly from Claim 4.3 and the following lemma.

Lemma 4.4. $b_{n, k}^{+} \leq f_{\mathcal{K}_{\mathcal{S}_{k}}}^{+}$and $b_{n, k}^{-} \leq f_{\mathcal{K}_{\mathcal{S}_{k}}}^{-}$hold for every $k \geq 3$ and sufficiently large $n$. 
Proof: Throughout this proof we assume that Enforcer's bias $b$ satisfies $b \leq \frac{1}{4} n^{\frac{k}{k-1}}$. For simplicity, we first assume that $b$ also satisfies $b=\omega(n)$. We propose a strategy for Enforcer which is very similar to the proposed strategy in the monotone game. However, some modifications are inevitable. One major difference between the two versions of the game is that the appearance of one threat (recall that a threat is a free edge incident to a vertex of $A$-degree $k-1$ ) does not secure Enforcer's win, so he has to make sure that $r$ threats appear before the last round. We therefore say that the game is in a winning position if either the maximal degree in Avoider's graph is at least $k$ or there exist at least $r$ threats. Since Enforcer cannot increase Avoider's degrees or the number of threats, Enforcer wins the game if and only if the game is in a winning position after Avoider's penultimate move. For convenience we denote $\ell=\left\lceil\left(\begin{array}{l}n \\ 2\end{array}\right) /(b+1)\right\rceil-1$ (i.e. the game lasts exactly $\ell+1$ rounds).

Another difference between the two versions of the game is that in the strict game Enforcer cannot maintain the property that every free edge is incident to at least one vertex isolated in his graph. However, he is able to maintain a partition $V=I \cup C$ (where $I_{i}$ and $C_{i}$ denote the respective sets at the end of the $i$ th round) with some similar properties. The exact construction of the sets $I$ and $C$ will be explained shortly. Initially, as in the monotone game, $I_{0}=V$ and $C_{0}=\emptyset$. Once again we denote by $T(i):=$ $\frac{1}{\left|I_{i}\right|} \sum_{v \in I_{i}} d_{A_{i}}(v)$ the average $A$-degree of the vertices in $I_{i}$ at the end of round $i$.

Enforcer's strategy involves dividing the course of the game into two stages. The game begins at Stage I; for every $0<i<\ell$, if the game is in a winning position before Enforcer's $i$ th move then Stage I is over and Enforcer immediately proceeds to Stage II. Otherwise, he keeps playing in Stage I. If before Enforcer's $\ell$ th move the game is still in Stage I, he proceeds to Stage II even if the game is not in a winning position. So, for some $1 \leq i \leq \ell$ Avoider's $i$ th move is the last move in Stage I and Enforcer's $i$ th move is the first move in Stage II. In each stage, Enforcer plays as follows.

Stage I: For every $i \geq 0$ such that Enforcer plays his $(i+1)$ st move in this stage, let $v_{1}^{(i)}, \ldots, v_{\left|I_{i}\right|}^{(i)}$ be an enumeration of the vertices in $I_{i}$ for which $d_{A_{i+1}}\left(v_{j}^{(i)}\right) \leq d_{A_{i+1}}\left(v_{j+1}^{(i)}\right)$, and if $d_{A_{i+1}}\left(v_{j}^{(i)}\right)=$ $d_{A_{i+1}}\left(v_{j+1}^{(i)}\right)$ then $d_{E_{i}}\left(v_{j}^{(i)}\right) \geq d_{E_{i}}\left(v_{j+1}^{(i)}\right)$, for $1 \leq j<\left|I_{i}\right|$. Let $I_{i, j}=\left\{v_{1}^{(i)}, \ldots, v_{j}^{(i)}\right\}$ and let $s_{i}$ be the largest integer such that the number of free edges inside $C_{i} \cup I_{i, s_{i}}$ is at most $b$. Every move consists of two parts.

In the first part of every move Enforcer claims all the free edges inside $C_{i} \cup I_{i, s_{i}}$ and he moves $I_{i, s_{i}}$ from $I$ to $C$, i.e. defines $C_{i+1}:=C_{i} \cup I_{i, s_{i}}$ and $I_{i+1}:=I_{i} \backslash I_{i, s_{i}}$.

For the second part of every move, let $l_{i+1}$ denote the number of edges Enforcer must claim in order to complete his $(i+1)$ st move. For every vertex $v \in\left\{v_{s_{i}+1}^{(i)}, \ldots, v_{s_{i}+4 k}^{(i)}\right\}$, Enforcer claims either $\left\lfloor\frac{l_{i+1}}{4 k}\right\rfloor$ or $\left\lceil\frac{l_{i+1}}{4 k}\right\rceil$ arbitrary free edges $v u$ such that $u \in C_{i+1}$, to get a total of $l_{i+1}$ edges, thus completing his move. We say that these edges are attached to $v$.

Stage II: In every step of every move in this stage, Enforcer claims an arbitrary edge which is not a threat if such an edge exists, and an arbitrary threat otherwise. He no longer maintains the partition $V=I \cup C$.

First we show that Enforcer can follow the proposed strategy. This is obvious for Stage II and for the first part of every move in Stage I. Assume now that Enforcer is trying to play the second part of his $i$ th move in Stage I for some $i>0$, after playing successfully all his previous moves according to the proposed strategy, including the first part of the $i$ th move. In particular, the partition $V=I_{i} \cup C_{i}$ has been 
determined. It is easy to see that at this point, exactly as in the monotone game, every free edge has at least one endpoint in $I_{i}$. Therefore, if there are less than $4 k$ vertices in $I_{i}$ then there are only $O(n)=o(b)$ free edges remaining (by our assumption $b=\omega(n)$ ), which implies $i \geq \ell$, in contradiction to the assumption that Enforcer is playing his $i$ th move in Stage I.

Hence, it only remains to show that Enforcer will be able to attach enough edges to every vertex among the first $4 k$ of $I_{i}$. Observe that $l_{i}<\left|C_{i}\right|$ by definition of $s_{i-1}$ and that $\left|C_{j}\right|=\omega(1)$ for every $j>0$. The following claim shows that Enforcer can indeed follow the second part of his moves in Stage I.

Claim 4.5. Throughout Stage I there are at least $\left(\frac{3}{4}-o(1)\right)|C|$ free edges between every vertex in I and $C$.

Proof: Since $d_{A}(v)<k$ for every $v \in V$ throughout Stage I, it suffices to show that for every round $i$ in this stage, $d_{E_{i}}(v) \leq\left(\frac{1}{4}+o(1)\right)\left|C_{i}\right|$ for every $v \in I_{i}$.

Let $v \in I_{i}$ be a vertex that was touched by Enforcer in his $i$ th move. If Avoider does not touch $v$ in his $(i+1)$ st move, then in every proper enumeration of the vertices in $I_{i}$ before Enforcer's $(i+1)$ st move, $v$ will be among the first $4 k$ vertices. Indeed, let $u$ be a vertex that was placed after the first $s_{i}+4 k$ vertices of $I_{i-1}$ in the $i$ th enumeration. By the properties of the enumeration and our assumption we get $d_{A_{i+1}}(v)=d_{A_{i}}(v) \leq d_{A_{i}}(u) \leq d_{A_{i+1}}(u)$. In case of equality we get $d_{E_{i}}(v)>d_{E_{i-1}}(v) \geq d_{E_{i-1}}(u)=$ $d_{E_{i}}(u)$. Enforcer will then add $v$ to $C_{i+1}$ since $b>4 k n$. So, $v$ remains in $I$ only if Avoider touches it in his $(i+1)$ st move and therefore every vertex can have edges attached to it by Enforcer in at most $k$ rounds.

Now consider a vertex $v \in I_{i}$ for some $i>0$ (the claim is trivial for $i=0$ ). Since $l_{j+1}<\left|C_{j+1}\right| \leq\left|C_{i}\right|$ for every $j<i$, the number of edges attached to $v$ cannot be more than $k\left\lceil\frac{\left|C_{i}\right|}{4 k}\right\rceil=\left(\frac{1}{4}+o(1)\right)\left|C_{i}\right|$.

We now wish to examine the course of the game in which Avoider plays according to some fixed strategy and Enforcer plays according to the proposed strategy, in order to obtain a sufficient condition for Enforcer's win, thus proving the lemma. Note that if Enforcer plays according to Stage II of the strategy at any move before his $\ell$ th, he wins the game. Assume, then, that this does not happen. It is immediate to observe that some properties hold exactly as in the monotone game.

Observation 4.6. The following properties hold throughout Stage I.

(i) After every move, by either player, every free edge has at least one endpoint in I.

(ii) Enforcer has no edges inside $I$.

(iii) $T(0)=0$ and $T(i+1)>T(i)$.

The following claim is the strict analogue of Claim 4.2, showing that as $I$ gets smaller, the average $A$-degree of its vertices becomes larger.

Claim 4.7. For every $0 \leq j \leq k-2$ the following holds. If for some $i$ Enforcer plays his ith move according to Stage I of his strategy and $0<\left|I_{i}\right|<\frac{9}{10} n\left(\frac{n}{2 b}\right)^{j}$, then $T(i) \geq j$.

Proof: We prove the claim by induction on $j$. The claim trivially holds for $j=0$, as $T(i) \geq 0$ for every $i$. Suppose now for contradiction that for some $1 \leq j \leq k-2$, the claim holds for $j-1$, but not for $j$. Then there exists an integer $i$ such that $0<\left|I_{i}\right|<\frac{9}{10} n\left(\frac{n}{2 b}\right)^{j}$, but $T(i)<j$. 
As in the proof of Claim 4.2, we denote by $i_{0} \leq i$ the minimal index such that $T\left(i_{0}\right) \geq j-1$. Since the integer-valued weight function $W(s):=\sum_{v \in I_{s}}\left(d_{A_{s}}(v)-(j-1)\right)$ is non-negative for $i_{0}$, and is strictly increasing for $s \leq i$, we conclude that $i-i_{0}+1 \leq W(i)+1 \leq\left|I_{i}\right|$ and thus between rounds $i_{0}$ and $i$ Enforcer has claimed at most $b\left|I_{i}\right|<\frac{9}{20} n^{2}\left(\frac{n}{2 b}\right)^{j-1}$ edges.

We now show that according to his strategy Enforcer had to claim more edges than that during these rounds. We distinguish between the following cases:

For $j=1$, Avoider could not have claimed any edge inside $C_{i}$, so the number of edges Enforcer had to claim is at least $\left(\begin{array}{c}\left|C_{i}\right| \\ 2\end{array}\right)=(1-o(1))\left(\begin{array}{c}n \\ 2\end{array}\right)>\frac{9}{20} n^{2}$.

For $j>1$, note that $i_{0}>0$. If $\left|I_{i_{0}-1}\right|=\Theta(n)$ it means that Enforcer claimed a quadratic number of edges in the specified rounds (all edges inside $I_{i_{0}-1} \backslash I_{i}$, except at most $k n$ edges that could have been claimed by Avoider). On the other hand, if $\left|I_{i_{0}-1}\right|=o(n)$, note that Enforcer had to claim all the edges between $I_{i_{0}-1} \backslash I_{i}$ and $C_{i_{0}-1}$ that were free before round $i_{0}$, except at most $k n$ edges. By Claim 4.5 and the induction hypothesis, the number of these edges is at least

$$
\left(\frac{9}{10} n\left(\frac{n}{2 b}\right)^{j-1}-\frac{9}{10} n\left(\frac{n}{2 b}\right)^{j}\right)\left(\frac{3}{4}-o(1)\right) n>\frac{9}{20} n^{2}\left(\frac{n}{2 b}\right)^{j-1} .
$$

In either case, we get a contradiction.

Let $g$ be the maximal index such that $\left|I_{g}\right|>0$ and $T(g)<k-2$ (there exists such an index since both inequalities hold for $g=0$ ). The next claim shows that as the game goes on after the $g$ th round, more and more vertices of $A$-degree $k-1$ appear in the graph.

Claim 4.8. For every $i \geq 0$, after Avoider's $(g+1+i)$ th move either Avoider's graph contains an $\mathcal{S}_{k}$ or there are at least $i$ vertices in $I_{g+i}$ of A-degree $k-1$.

Proof: Let $v_{1}, \ldots, v_{t}$ denote the vertices of $I_{g+1}$ with $A$-degree at most $k-3$ after the $(g+1)$ st round, and let $m=\sum_{i=1}^{t}\left(k-2-d_{A_{g+1}}\left(v_{i}\right)\right)$. If Avoider has not yet created an $\mathcal{S}_{k}$, all vertices have $A$-degree at most $k-1$, thus after the $(g+1)$ st round there are at least $m$ vertices in $I_{g+1}$ with $A$-degree $k-1$, as the average $A$-degree in $I_{g+1}$ is at least $k-2$ by definition of $g$. Since all free edges have at least one endpoint in $I$, as long as Enforcer removes from $I$ only vertices of $A$-degree at most $k-2$, in every round after the $(g+1+m)$ th the number of vertices of $A$-degree $k-1$ is increased, or an $\mathcal{S}_{k}$ appears in Avoider's graph. If Enforcer removes from $I$ a vertex of $A$-degree at least $k-1$, then if the maximal degree in Avoider's graph is $k-1$ at that point, it will be increased to $k$ in Avoider's subsequent move (if such a move exists).

By Claim 4.7 we get $\left|I_{g}\right| \geq \frac{9}{10} n\left(\frac{n}{2 b}\right)^{k-2}$. Hence, either $I_{g}$ is of linear order and then $\left|F_{g}\right|=\Theta\left(n^{2}\right)$ (since at most $k|I|$ edges can be claimed inside $I$ throughout Stage I), or $\left|C_{g}\right|=(1-o(1)) n$ and then by Claim 4.5 we get:

$$
\left|F_{g}\right| \geq\left(\frac{3}{4}-o(1)\right)\left|I_{g}\right| n \geq(1-o(1)) \frac{27}{40} n^{2}\left(\frac{n}{2 b}\right)^{k-2} .
$$

Thus, the number of rounds remaining in the game after the $g$ th round satisfies

$$
\ell+1-g=\left\lceil\frac{\left|F_{g}\right|}{b+1}\right\rceil \geq(1-o(1)) \frac{27}{20} n\left(\frac{n}{2 b}\right)^{k-1} .
$$


Using our assumption $b \leq \frac{1}{4} n^{\frac{k}{k-1}}$, a simple calculation yields (as $k \geq 3$ ):

$$
n=\frac{n^{k}}{(2 b)^{k-1}}\left(\frac{2 b}{n}\right)^{k-1} \leq \frac{1}{4} \frac{n^{k+1}}{(2 b)^{k-1}} .
$$

By Claim 4.8 we have that after Avoider's $\ell$ th move there are at least $\ell-g-1$ vertices of $A$-degree $k-1$ in $I_{\ell-1}$. Since every such vertex creates at least $\left(\frac{3}{4}-o(1)\right) n$ unique threats, by using (1) and (2) we get that the number of threats after Avoider's $\ell$ th move is at least

$$
\left((1-o(1)) \frac{27}{20} n\left(\frac{n}{2 b}\right)^{k-1}-2\right)\left(\frac{3}{4}-o(1)\right) n \geq \frac{n^{k+1}}{(2 b)^{k-1}}-\frac{3}{2} n \geq \frac{5}{8} \frac{n^{k+1}}{(2 b)^{k-1}}
$$

As already mentioned, if the maximal degree in $A_{\ell}$ is less than $k$, then Enforcer wins if and only if there are at least $r$ threats after Avoider's $\ell$ th move.

By definition of $f^{+}$and $b_{n, k}^{+}$, and since $b_{n, k}^{+}=\omega(n)$ by Claim 4.3 , it is clear that $b_{n, k}^{+} \leq f_{\mathcal{K}_{\mathcal{S}_{k}}^{+}}$. However, in order to show that $b_{n, k}^{-} \leq f_{\mathcal{K}_{\mathcal{S}_{k}}}$ holds as well, we must show in addition that Enforcer has a winning strategy if $b=O(n)$. Indeed, if $b=o(n)$ Enforcer wins no matter how he plays since Avoider will have $\omega(n)$ edges in his final graph, so assume $b=\Theta(n)$. Enforcer does the following: before the game starts he chooses an arbitrary set $U \subseteq V$ of size $|U|=(2 b)^{\frac{k}{k+1}}<n$, and in each step he claims some arbitrary free edge with at least one endpoint outside $U$ until he can no longer do so, i.e. until all free edges lie completely inside $U$. Then he pretends to start a new game on $n^{\prime}=(2 b)^{\frac{k}{k+1}}$ vertices with bias $b=\frac{1}{2} n^{\prime \frac{k+1}{k}}$ according to the strategy for the case $b=\omega(n)$. This is not exactly a new game because there may be some edges inside $U$ already claimed by Avoider, and the "new" game may start during Enforcer's move. However, since Avoider can claim only a constant number of edges incident to each vertex, and since Enforcer makes at most $b$ additional steps before Avoider's first move in the new game, these factors have no significant effect. They only affect the case $j=1$ in the proof of Claim 4.7, and it is easy to see that the analysis there is still valid. The number of free edges before the last round (i.e. $r\left(n^{\prime}, b\right)$ ) is also affected, but since $b \leq b_{n^{\prime}, k}^{-}$Enforcer wins regardless of the exact value of $r$.

\section{An application of Fact 2.1}

We mentioned in the introduction that Bednarska-Bzdȩga in [2] obtained bounds on the different threshold biases for general $H$-games. For every fixed graph $H$ with at least two edges, let

$$
\begin{gathered}
m(H)=\max _{F \subseteq H: v(F) \geq 1} \frac{e(F)}{v(F)} ; \quad m^{\prime}(H)=\max _{F \subseteq H: v(F) \geq 1} \frac{e(F)-1}{v(F)} ; \\
m_{2}(H)=\max _{F \subseteq H: e(F) \geq 2} \frac{e(F)-1}{v(F)-2},
\end{gathered}
$$

where $v(F)$ and $e(F)$ denote the number of vertices and number of edges of the subgraph $F$, respectively. Bednarska-Bzdȩga proved the following ([2], Theorems 1.9 and 1.10):

(i) $f_{\mathcal{K}_{H}^{m o n}}^{\text {mon }}=O\left(n^{1 / m^{\prime}(H)}\right)$ and $f_{\mathcal{K}_{H}}^{+}=O\left(n^{1 / m^{\prime}(H)}\right)$; 
(ii) $f_{\mathcal{K}_{H}}^{\text {mon }}=\Omega\left(n^{1 / m_{2}(H)} / \ln n\right)$ and $f_{\mathcal{K}_{H}}^{-}=\Omega\left(n^{1 / m_{2}(H)} / \ln n\right)$;

(iii) $f_{\mathcal{K}_{H}}=O\left(n^{1 / m(H)} \ln n\right)$ always holds, and $f_{\mathcal{K}_{H}}=O\left(n^{1 / m(H)}\right)$ holds for infinitely many values of $n$.

In the proof of ( $i i i)$ she uses her general criterion for Avoider's win and shows that for $f=c n^{1 / m(H)}$ (for some constant $c \geq 1$ ), if $r\left(n, f^{\prime}\right)>f$, then Avoider wins the strict $\left(1: f^{\prime}\right) H$-game played on the edges of $K_{n}$. She uses two number theoretical facts to show that there always exists such an $f^{\prime}$ satisfying $f^{\prime} \leq 4 m(H) f \ln f$, and that for infinitely many values of $n$ there exists such an $f^{\prime}$ satisfying $f^{\prime} \leq 2 f$. She only considers the case $m(H)>1 / 2$ (the other case is trivial), and so by applying Fact 2.1] we obtain the following corollary.

Corollary 5.1. If $m(H) \leq 1$ then $f_{\mathcal{K}_{H}}=O\left(n^{1 / m(H)}\right)$.

That is, we obtain the better bound for all $n$ in this case. Note that $m(H) \leq 1$ if and only if $H$ is a graph in which every connected component contains at most one cycle.

Our results show that these bounds are far from being tight for the star game, except for the "improved" upper bound on $f_{\mathcal{K}_{H}}^{-}$given in Corollary 5.1 where we got exactly the same result. We proved the bound for $f_{\mathcal{K}_{\mathcal{S}_{k}}}^{-}$in our paper explicitly anyway, since it is straightforward to obtain by using Fact 2.1 and our other arguments. The gaps in $(i)$ and $(i i)$ are not very surprising, as these bounds are valid for every fixed graph $H$. However, at least the upper bound on $f_{\mathcal{K}_{H}}^{\text {mon }}$ cannot be improved in general, since it is tight for the case $H=K_{3}$. In addition, the constant exponent $1 / m(H)$ in both bounds of (iii), as well as in Corollary 5.1 cannot be improved in general, because for $H=P_{3}$ we have $f_{\mathcal{K}_{P_{3}}}^{-}=\Omega\left(n^{1 / m(H)}\right)$, as observed by Bednarska-Bzdęga herself in [2]. In this paper we provided an infinite family of graphs for which this bound is tight.

\section{Concluding remarks and open problems}

In Section 4 we propose a very natural strategy for Enforcer in the $k$-star game to enforce the appearance of a vertex of large degree in Avoider's graph. In [5], Gebauer and Szabó use a very similar approach; they study the change of the average degree in Breaker's graph over some subset of vertices during the game and show that it cannot get too large, and thus Maker's graph has a large minimum degree. So, enforcing a large average degree (and thus the maximal degree) in Avoider's graph over a subset of vertices complements in a way the method of Gebauer and Szabó.

In this paper we show that for every sufficiently large $n$ and every $k \geq 3$, the threshold biases $f_{\mathcal{K}_{\mathcal{S}_{k}}}$ and $f_{\mathcal{K}_{\mathcal{S}_{k}}}^{+}$are not of the same order, thus supporting the conjecture of Hefetz et al. from [6]. In that paper they also showed that $f_{\mathcal{K}_{H}}^{\text {mon }}$ and $f_{\mathcal{K}_{H^{-}}}^{-}$are of the same order for $H=K_{3}$; we showed the same for $H=\mathcal{S}_{k}$. Observe that $H^{-}=P_{3}$ for both $H=K_{3}$ and $H=\mathcal{S}_{3}$, and so $f_{\mathcal{K}_{H}}^{\text {mon }}$ is of the same order in both cases. It would be interesting to further investigate the relation between the monotone $H$-games and the strict $H^{-}$-games and to determine whether indeed there is a connection between the two. Note that for a general graph $H$, the graph $H^{-}$is not uniquely determined (unlike the $K_{3}$ and $\mathcal{S}_{3}$ cases) and so for different choices of $H^{-}$there are different outcomes. Therefore, choosing the "correct" $H^{-}$must also be considered.

In Theorem 1.1 we provided the bounds for $f_{\mathcal{K}_{\mathcal{S}_{k}}}^{\text {mon }}, f_{\mathcal{K}_{\mathcal{S}_{k}}}^{+}$and $f_{\mathcal{K}_{\mathcal{S}_{k}}}^{-}$that are tight up to a constant factor. We could actually get some better (tighter) bounds - for example, by refining Avoider's strategy we could 
show that the constant in the upper bound in all three cases is $1+\varepsilon$, for any $\varepsilon>0$, rather than $2-$ but since we could not close the gap completely we decided to provide slightly weaker bounds with simpler proofs. It would be nice to determine the exact values of $C_{1}, C_{2}$ and $C_{3}$ for which $f_{\mathcal{K}_{\mathcal{S}_{k}}^{m o n}}=C_{1} n^{\frac{k}{k-1}}$, $f_{\mathcal{K}_{\mathcal{S}_{k}}}^{+}=C_{2} n^{\frac{k}{k-1}}$ and $f_{\mathcal{K}_{\mathcal{S}_{k}}}^{-}=C_{3} n^{\frac{k+1}{k}}$. In addition, note that our results for the $k$-star game only hold for a constant $k$.

Let us comment on the case when $k=k(n)$ tends to infinity along with $n$. Clearly, as long as the bias $b$ satisfies $b \leq(1-\varepsilon) \frac{n}{k}$, Avoider is doomed as at the end of the game even the average degree of his graph will be larger than $k$. Thus $(1-o(1)) \frac{n}{k} \leq f_{\mathcal{K}_{\mathcal{S}_{k}}}, f_{\mathcal{K}_{\mathcal{S}_{k}}}^{+}, f_{\mathcal{K}_{\mathcal{S}_{k}}}^{\text {mon }}$ holds. On the other hand, Avoider could win when $b \geq(1+\varepsilon) \frac{n}{k}$ provided he could keep all degrees asymptotically the same. This kind of discrepancy games were studied first by Erdős, and the following general result of Beck tells us the order of magnitude of the threshold biases when $k=\omega(\log n)$. He considered the game of Balancer (playing with bias $p$ ) and Unbalancer (playing with bias $q$ ) in which they claim elements of a board $X$.

Theorem 6.1 (Theorem 17.5 in [1]). Let $\mathcal{F}$ be an arbitrary $N$-uniform hypergraph. Balancer and Unbalancer play the $(p: q)$ game: they alternate, Balancer takes $p$ new points and Unbalancer takes $q$ new points per move. Then Balancer, as the first player, can force that, at the end of the play, for every $A \in \mathcal{F}$, his part in $A$ is strictly between $\frac{p+\varepsilon}{p+q} N$ and $\frac{p-\varepsilon}{p+q} N$, where

$$
\varepsilon=\left(1+O\left(p q \sqrt{\frac{\log |\mathcal{F}|}{(p+q) N}}\right)\right) 2 p q \sqrt{\frac{\log |\mathcal{F}|}{(p+q) N}} .
$$

Avoider can use Balancer's strategy in the above game with $p=1, q \geq(1+\varepsilon) \frac{n}{k}, N=n-1$ and $|\mathcal{F}|=n$ with $\mathcal{F}$ consisting of the edge sets of the stars of $K_{n}$. An easy calculation shows that if $k=\omega(\log n)$, then $\varepsilon$ can be chosen to satisfy $\varepsilon=o(1)$. Thus we obtain $f_{\mathcal{K}_{\mathcal{S}_{k}}}, f_{\mathcal{K}_{\mathcal{S}_{k}}}^{+}=(1+o(1)) \frac{n}{k}$ for these values of $k$. The case $\omega(1)=k=O(\log n)$ remains open.

\section{Acknowledgements}

The research was initiated at the 4th Emléktábla Workshop held in Tihany, August 6-9, 2012. The authors would like to thank Asaf Ferber and Miloš Stojaković for their useful comments and suggestions. Also, the authors would like to express their gratitude to the referees whose thorough remarks greatly improved the paper. Furthermore, Fact 2.2 was pointed out by one of the referees along with the remark about the case $k \rightarrow \infty$ in the last section.

\section{References}

[1] J. Beck, Combinatorial Games: Tic-Tac-Toe Theory, Cambridge University Press, 2008

[2] M. Bednarska-Bzdȩga, Avoider-Forcer games on hypergraphs with small rank, Electronic Journal of Combinatorics, 21(1) (2014), P1.2.

[3] B. Bollobás, Random Graphs, Cambridge University Press, 2001.

[4] V. Chvátal and P. Erdős, Biased positional games, Annals of Discrete Mathematics 2 (1978), 221228. 
160 Andrzej Grzesik, Mirjana Mikalački, Zoltán Lóránt Nagy, Alon Naor, Balázs Patkós, Fiona Skerman

[5] H. Gebauer and T. Szabó, Asymptotic random graph intuition for the biased connectivity game, Random Structures and Algorithms, 35 (2009), 431-443.

[6] D. Hefetz, M. Krivelevich, M. Stojaković and T. Szabó, Avoider-Enforcer: The rules of the game, Journal of Combinatorial Theory Series A 117 (2010), 152-163.

[7] D. Hefetz, M. Krivelevich and T. Szabó, Avoider-Enforcer games, Journal of Combinatorial Theory Series A 114 (2007), 840-853.

[8] S. Janson, T. Łuczak, A. Ruciński, Random Graphs, John Wiley \& Sons, Inc., 2000.

[9] M. Krivelevich and T. Szabó, Biased positional games and small hypergraphs with large covers, Electronic Journal of Combinatorics, 15 (2008), R70.

[10] D. B. West, Introduction to Graph Theory, Prentice Hall, 2001. 